\title{
Prevalence and factors associated with tuberculosis treatment outcome among hazardous or harmful alcohol users in public primary health care in South Africa
}

\author{
Peltzer $\mathrm{K}^{1,2}$, Louw JS
}

1.HIV/AIDS, TB and STI (HAST) Research Programme, Human Sciences Research Council, Pretoria and Durban, South Africa.

2. Department of Psychology, University of Limpopo, Turfloop, South Africa

3. ASEAN Institute for Health Development, Mahidol University, Salaya, Thailand

\begin{abstract}
Background: Tuberculosis (TB) remains a chronic infectious disease with high morbidity and mortality.

Objective: The aim of this study was to assess the prevalence and associated factors of tuberculosis treatment failure, death and default among hazardous or harmful alcohol users.
\end{abstract}

Method: We conducted a prospective study with TB patients in 40 public health clinics in three districts in South Africa. All consecutively new tuberculosis and retreatment patients presenting at the 40 primary health care facilities with hazardous or harmful alcohol use were included in this study. Logistic regression was used to assess determinants of TB treatment failure, death and default.

Results: The findings of our study showed that $70 \%$ of TB patients were either cured or had completed their TB treatment by the end of 6 months. In multivariate analysis participants living in a shack or traditional housing (Odds Ratio=OR: 0.63, Confidence Interval=CI: 0.45-0.89), being a TB retreatment patient (OR: 1.61, CI: 1.15-2.26) and residing in the eThekwini district (OR: 1.82, CI: 1.27-2.58) were significant predictors of treatment failure, death and default.

Conclusion: A high rate of treatment failure, death and default were found in the TB patients. Several factors were identified that can guide interventions for the prevention of treatment failure, death and default.

Key words: Tuberculosis, treatment failure, default, determinants, primary care, South Africa African Health Sciences 2014;14(1):157-166 http://dx.doi.org/10.4314/ahs.v14i1.24

\section{Background}

Although there are numerous global efforts to control tuberculosis (TB), it remains a chronic infectious disease with high morbidity and mortality in several parts of the world. ${ }^{1,2,3}$ As the world's most frequent contagious disease, TB causes about 2 million deaths

a year, with more than 8 million people contractingthe disease every year. ${ }^{4,5}$ According to the $\mathrm{WHO}^{6}{ }^{6}$ an estimated 1.7 million people died from TB in 2009, with the highest number of TB related deaths being in the African region.

While modern TB treatment regimens are generally

\section{*Correspondence author: \\ Karl Peltzer \\ HAST, Human Sciences Research Council Private Bag X41, Pretoria 0001, South Africa \\ Email: KPeltzer@hsrc.ac.za}

associated with a good prognosis, ${ }^{7}$ factors such as bacterium characteristics, heterogeneity of patients' clinical characteristics, patient behaviour, quality of health care, HIV co-infection and multi-drug resistant tuberculosis (MDRTB) are known to influence TB treatment outcomes. ${ }^{2,8,9,10} \mathrm{~A}$ number of factors including age, male gender, delays in diagnosis and treatment, drug resistance, and co-morbid conditions including HIV co-infection, diabetes ${ }^{1}$, alcoholism, smoking, lower educational and income levels, unemployment, treatment for other concomitant diseases, and side effects of antiTB drugs ${ }^{11}$ have been associated with increased risk of death in patients with active tuberculosis. Various studies support a strong association between alcohol use, alcohol use disorders and TB. ${ }^{12-14}$ These studies show the pathogenic impact of alcohol on the immune system causing susceptibility to TB among drinkers. ${ }^{13}$ People that drink heavily show higher relapse rates, a 
higher probability of an unfavourable clinical course and a higher probability of experiencing the most destructive forms of TB. ${ }^{12,14} \mathrm{~TB}$ patients with an unsuccessful treatment outcome, especially due to treatment failure and default, are a public health concern as they are at risk for clinical deterioration and complications, can continue to be infectious to others, and are at risk of premature death from TB. ${ }^{9,12}$ TB treatment default, defined by the World Health Organization (WHO) as a treatment interruption of two consecutive months or more after at least one month on treatment, ${ }^{13}$ is among the factors that has been linked to TB related mortality, $45 \%$ in Vietnam, 27\% in Mexico, 28\% in South Africa and $54 \%$ in the USA. ${ }^{14}$ Various studies conducted in West Asia, Australia, Canada, Russia, Switzerland, and the USA had identified potential risk factors for TB treatment default. ${ }^{15-18}$

There seems to be strong evidence that the risk of death and default increases among HIV positive patients. ${ }^{12,19-}$ ${ }^{21}$ However, in addition to HIV co-infection being a risk factor, other predictive factors attributed to TB treatment default among $\mathrm{TB}$ patients are such risk factors as ignorance, travelling long way to treatment site, feeling better, side-effects, inadequate knowledge about TB, herbal medication use, low income, alcohol abuse, previous default, and the male sex. ${ }^{12,20,22}$ Alcohol abuse has also been associated with forgetting to take treatment (in $7.5 \%$ cases) and consequently defaulting. ${ }^{20}$

In 2007, the African continent had thelowest tuberculosis (TB) cure rate (54\%-74\%) compared to the global cure rate $(84 \%)$, primarily due to the lack of treatment adherence. $^{23}$ A low cure rate and a high treatment default rate provide opportunities for maintaining transmission, relapse or treatment failure, mortality and the development of resistant TB. ${ }^{22,24}$ Some studies have indicated that in sub-Saharan Africa poor public transport contributes to high treatment default. ${ }^{20,25}$

The aim of this study was to assess the prevalence and associated risk factors of tuberculosis treatment failure, death and default among hazardous or harmful alcohol users in selected health districts in South Africa.

\section{Methodology}

\section{Sampling and procedure}

This was a prospective study conducted with TB patients with alcohol problems in public health clinics in South Africa. Three provinces, in South Africa, with the highest TB caseload were selected for inclusion in the study. One district in each province $(\mathrm{N}=3)$ with the highest TB caseloads were consequently included. These districts were Siyanda in the Northern Cape Province, Nelson Mandela Metro in the Eastern Cape Province, and the e'Thekwini in KwaZulu-Natal Province. Clinics with a high TB caseload (based on statistics provided by the Department of Health) in each of the three study districts with a high burden of TB were included in the study. Within each of these three study districts 14 public primary health care facilities were selected based on the highest TB caseloads per clinic, and 2 clinics were excluded since they had no alcohol abusing patients so that 40 clinics remained, 14 in eThekwini, 13 in Nelson Mandela Metro and 13 in Siyanda. The type of health facility was a primary health care clinic or community health centre. The study catchment areas within the study health districts and randomization procedures enabled a broad coverage of major population groups.

TB patients were consecutively recruited and interviewed by trained research assistants over a period of six months. A health care provider who identified a new or retreatment TB patient (within one month of treatment initiation) informed the patient about the study and if interested, were referred to the research assistant. A research assistant asked for consent from patients to participate in the study. All new tuberculosis and retreatment patients (i.e. those patients who have been initiated or have been on anti-TB treatment for less than one month), males and females, 18 years and above who visited the primary health care facility were 
screened using the Alcohol Use Disorder Identification Test (AUDIT). Those who scored 8 or more for men and 7 or more for women on the AUDIT questionnaire after the screening were included in this study. Research assistants conducted follow-up interviews at 6 months following baseline assessment at scheduled clinic visits. The research assistants were all individuals with a matric or Grade 12 qualification living in the communities where the study clinics were located and who spoke the predominant languages, namely English, Afrikaans, $\mathrm{i}-\mathrm{Zulu}$, i-Xhosa and Tswana, in the respective areas. They all attended a 3-day training in questionnaire administration, research procedures and ethics upon which they received a certificate for successful completion.

In addition, medical file information was collected for HIV and TB treatment status and outcome. Nonattenders were followed up by telephone and home visits arranged as necessary. Ethical approval was obtained from the Human Sciences Research Council Research Ethics Committee (Protocol REC No.1/16/02/11). The Department of Health in South Africa has also provided approval for this study.

\section{Measures}

The outcome was the successful TB response, classified by $\mathrm{WHO}$ as cured or treatment completed (versus treatment failure, defaulted, died or transferred out to another health facility. ${ }^{13}$ This was assessed by inspection of medical records. Further, patients who previously had not completed their TB treatment were asked as to why they had stopped TB treatment.

Socioeconomic characteristics assessed were age, gender, educational level, marital status, income, employment status, dwelling characteristics and residential status. Poverty was assessed with 5 items on the availability or non-availability of shelter, fuel or electricity, clean water, food and cash income in the past week. Response options ranged from 1="Not one day" to $4=$ "Every day of the week". Poverty was defined as higher scores on non-availability of essential items. The total score ranged from 5 to 20 with 20 indicative of the 'highest level of poverty'. The categories of poverty, therefore, are as follows: $5=$ low, $6-12=$ medium and $13-20=$ high. Cronbach alpha for the poverty index in this study was 0.89 .

Perceivedgeneral health: Participants were asked, "In general, would you say your health is: excellent, very good, good, fair or poor?" This measure was categorized based on participant response (very good = excellent/very good, good, and poor $=$ fair/poor).

\section{Chronic conditions:}

Two questions were asked to determine whether participants had chronic conditions and taking medications for their chronic conditions. The questions asked were "Has a doctor or nurse or health worker at a clinic or hospital ever told you that you have or have had any of the following conditions?" They were given a list of chronic conditions to choose from..$^{26,27}$ Response options were "yes" or "no".

The 10-item Kessler Psychological Distress Scale (K-10): was used to measure global psychological distress, including significant pathology which does not meet formal criteria for a psychiatric illness. ${ }^{28,29}$ The frequency with which each of the items was experienced was recorded using a five-point Likert scale ranging from "none of the time" to "all the time". This score was then summed with increasing scores reflecting an increasing degree of psychological distress. We examined the K-10 scale used as a binary variable, comparing scores of 10-15 versus 16 or more. The internal reliability coefficient for the K-10 in this study was alpha $=0.92$.

Alcohol consumption: The 10-item Alcohol Disorder Identification Test (AUDIT) ${ }^{30}$ assesses the alcohol consumption level (3 items), symptoms of alcohol dependence ( 3 items), and problems associated with alcohol use (4 items). Responses to items in the AUDIT are rated on a 4-point Likert scale from 0 to 4, for a maximum score of 40 points. Higher AUDIT scores 
indicate more severe levels of risk; scores 8 indicate a tendency to problematic drinking. Cronbach alpha for the AUDIT in this sample was 0.92, indicating excellent reliability. Hazardous drinking is defined as a quantity or pattern of alcohol consumption that places patients at risk for adverse health events, while harmful drinking is defined as alcohol consumption that results in adverse events (e.g., physical or psychological harm). ${ }^{31}$

Tobacco use: Two questions were asked about the use of tobacco products. (a) Do you currently use one or more of the following tobacco products (cigarettes, snuff, chewing tobacco, cigars, etc.)? Response options were 'yes' or 'no'. (b) In the past month, how often have you used one or more of the following tobacco products (cigarettes, snuff, chewing tobacco, cigars, etc.) Response options were once or twice, weekly, almost daily and daily.

\section{Data Analysis}

Data was analyzed using the IBM Statistical Package for the Social Sciences (SPSS) for Windows software application programme version 19.0. Frequencies, means, standard deviations, were calculated to describe the sample. Data were checked for normality distribution and outliers. Associations of TB treatment failure, death and default, sociodemographic and health variables were identified using logistic regression analyses. Following the univariate regression, a multivariable regression model was constructed. Independent variables from the univariate analyses were entered into the multivariable model if significant at $\mathrm{P}<0.05$ level. The $\mathrm{R}^{2}$ is presented to describe the amount of variance explained by the multivariable model. Probability below 0.05 was regarded as statistically significant.

\section{Results}

\section{Sample characteristics}

We identified 1196 TB patients across 40 primary care clinics. These patients were screened for alcohol and tested positive for the AUDIT. The participants were followed up at 6 months and assessed for TB treatment outcomes. 1049 (88\%) were followed-up and 12\% could not be followed up due to various reasons including misplaced fieldworkers codebooks, the incorrect recording of names, names that could not be matched with the clinic register and misplaced clinic registers.

The total sample at baseline comprised of 1196 participants with $48 \%$ between the age of $18-34$ years, $28 \%$ between the age of $35-44$ years and $24 \%$ were participants over 45 years of age. The majority of the total sample $(70 \%)$ was cured or completed their TB treatment and this rate was more for females compared to males. Thirty percent of the sample had treatment failure, died or defaulted on their treatment.

The majority of participants did not have a grade 12 or higher educational attainment $(88 \%)$, however only $21.8 \%$ scored high on the poverty index. Most of the participants relied on family contributions (38.2\%) followed by those who had a formal salary $(24.3 \%)$ and social grants (20.8\%), while $16.7 \%$ had no form of income. The participants who had a formal salary and those with no income $30.8 \%$ and $39.1 \%$, respectively), experienced more treatment failure, default and death compared to those who either relied on family contributions or social grants $(28.9 \%$ and $28.4 \%$, respectively). A third of the retreatment patients $(32.2 \%)$ who had treatment failure, defaulted or died, had reported an HIV positive status. Forty six percent of the sample perceived their health status as fair or poor. Fewer participants $(26.4 \%)$ with three or more chronic conditions experienced treatment failure, default and death compared to those who reported two, one or no other chronic conditions $(33.9 \%, 32.5 \%$ and, $29.4 \%$, respectively). 
TABLE 1

Associations with TB treatment failure, death and default for all TB patients (new and retreatment)

Table 1: Sample characteristics at baseline ( $<4$ weeks TB treatment) $(\mathrm{N}=1196)$

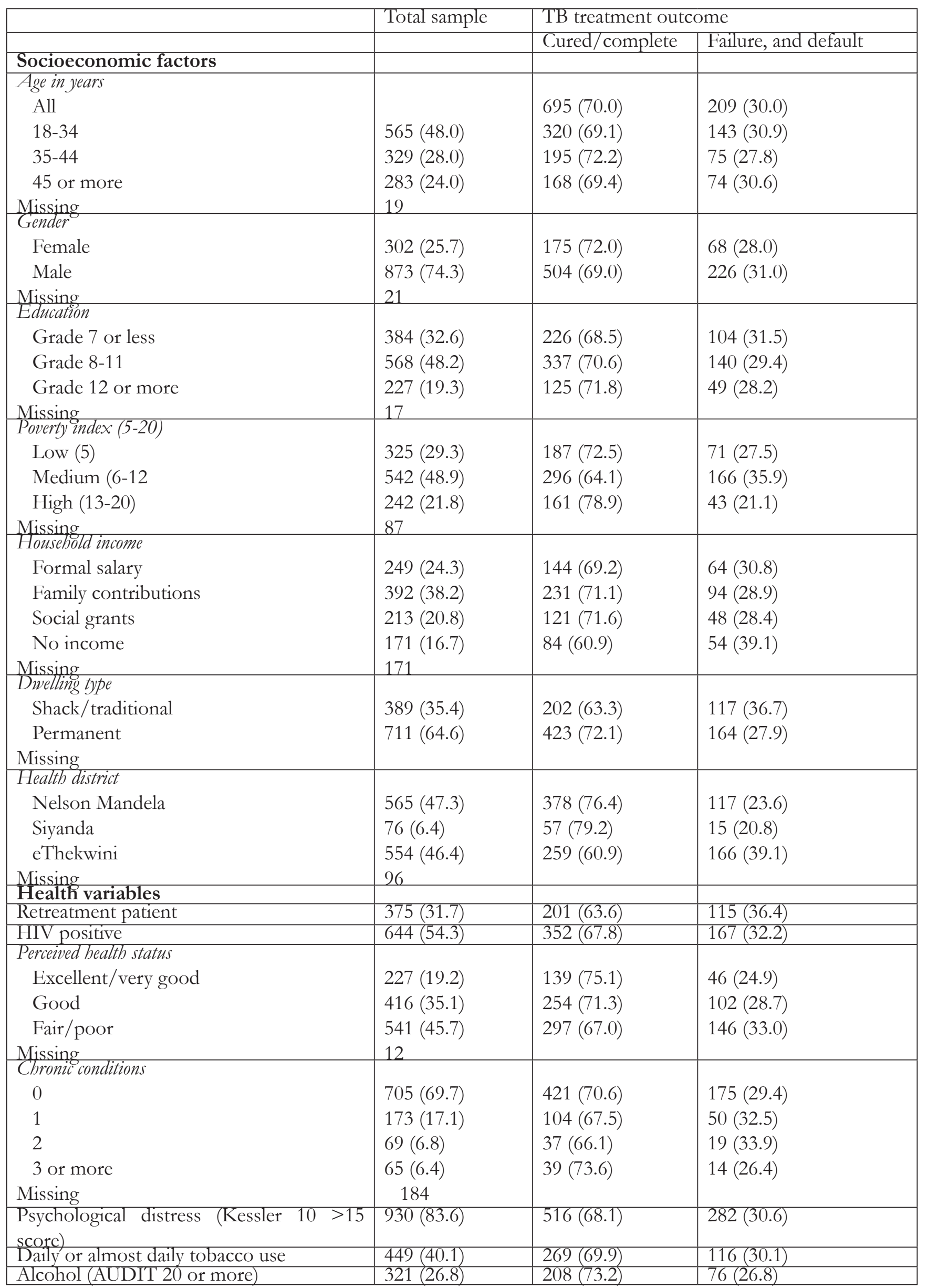


In multivariate analysis living in a shack or traditional district and being a retreatment patient were significant dwelling, patients from clinics in the eThekwini health predictors of treatment failure, death and default.

TABLE 2

Table 2: Regression model with TB treatment failure, death and default for all TB patients (new and retreatment)

\begin{tabular}{|c|c|c|}
\hline Socioeconomic factors & $\begin{array}{l}\text { Crude Odds Ratio }(95 \% \\
\mathrm{CI})\end{array}$ & $\begin{array}{l}\text { Adjusted Odds Ratio } \\
(95 \% \mathrm{CI})^{a}\end{array}$ \\
\hline Age & & \\
\hline $18-34$ & 1.00 & 1.00 \\
\hline $35-44$ & $0.86(0.62-1.20)$ & $0.90(0.62-1.32)$ \\
\hline 45 or more & $0.99(0.70-1.38)$ & $0.91(0.62-1.35)$ \\
\hline Gender & & \\
\hline Female & 1.00 & 1.00 \\
\hline Male & $1.15(0.84-1.59)$ & $1.17(0.80-1.72)$ \\
\hline Education & & \\
\hline Grade 7 or less & 1.00 & --- \\
\hline Grade 8-11 & $0.90(0.67-1.22)$ & \\
\hline Grade 12 or more & $0.85(0.57-1.28)$ & \\
\hline Poverty index & & \\
\hline Low & 1.00 & 1.00 \\
\hline Medium & $1.48(1.06-2.06)^{*}$ & $1.27(0.87-1.85)$ \\
\hline Hioh & $0.70(0.46-1.09)$ & $0.73(0.43-1.23)$ \\
\hline Housebold income & & \\
\hline Formal salary & 1.00 & --- \\
\hline Family contributions & $0.92(0.63-1.34)$ & \\
\hline Social grants & $0.89(0.57-1.39)$ & \\
\hline No income & $1.45(0.92-2.27)$ & \\
\hline Dwelling type & & \\
\hline Shack/traditional & 1.00 & 1.00 \\
\hline Permanent & $0.67(0.50-0.90) * *$ & $0.63(0.45-0.89)^{* *}$ \\
\hline Health district & & \\
\hline Nelson Mandela & 1.00 & 1.00 \\
\hline Siyanda & $0.85(0.46-1.56)$ & $0.76(0.38-1.51)$ \\
\hline eThekwini & $2.07(1.56-2.75) * * *$ & $1.81(1.27-2.58) * * *$ \\
\hline Health variables & & \\
\hline Retreatment patient & $1.54(1.15-2.04)^{* *}$ & $1.61(1.15-2.26)^{* *}$ \\
\hline HIV positive & $1.25(0.95-1.64)$ & --- \\
\hline Perceived health status & & \\
\hline Excellent/very good & 1.00 & 1.00 \\
\hline Good & $1.21(0.81-1.82)$ & $0.91(0.56-1.48)$ \\
\hline Fair/poor & $1.49(1.01-2.19)^{*}$ & $1.04(0.65-1.65)$ \\
\hline Chronic conditions & & \\
\hline 0 & 1.00 & --- \\
\hline 1 & $1.16(0.79-1.69)$ & \\
\hline 2 & $1.24(0.69-2.21)$ & \\
\hline 3 or more & $0.86(0.46-1.63)_{*}$ & \\
\hline Psychological distress (Kessler $10>15$ score) & $1.47(1.00-2.16)^{*}$ & $1.31(0.85-2.02)$ \\
\hline Daily or almost daily tobacco use & $0.97(0.74-1.29)$ & --- \\
\hline Alcohol (AUDIT 20 or more vs. $7 / 8-19$ ) & $0.80(0.59-1.09)$ & --- \\
\hline
\end{tabular}


Qualitative responses as to why patients defaulted on their treatment In total 106 patients responded with reasons as to why they had previously stopped TB medication,which were mainly significant health improvement, structural and psychosocial including alcohol problems (Table 3).

Table 3: Qualitative responses as to why patients defaulted on their TB treatment $(N=106)$

\begin{tabular}{|l|l|}
\hline Qualitative responses & $\%$ \\
\hline 1. Feeling Well (after talking to a nurse and three months after treatment) & 17.4 \\
\hline $\begin{array}{l}\text { 2. Only did 3 months of the first 6 months thereafter stopped because of symptoms, } \\
\text { was clean, no reason, just stopped }\end{array}$ & 15.9 \\
\hline 3 Changed jobs, moved location & 14.5 \\
\hline 4. Structural (no money to go to hospital/clinic, buy food, too far to clinic) & 13.0 \\
\hline $\begin{array}{l}\text { 5. Got tired of taking medications } \\
\text { 6. Misunderstanding with the health care provider, could not get a letter from where I } \\
\text { was diagnosed to where I was referred }\end{array}$ & 11.0 \\
\hline 7. An alcohol problem & 9.1 \\
\hline 8. Medication side effects (ear problem, vomiting) & 3.5 \\
\hline 9. Family problems, e.g. brother died & 2.9 \\
\hline 10. Imprisoned, arrested & 2.9 \\
\hline
\end{tabular}

\section{Discussion}

The findings of our study showed that the majority of new tuberculosis and retreatment patients $(70 \%)$ were either cured or had completed their TB treatment by the end of 6 months. Of concern though is the notable finding that $30 \%$ of TB patients had treatment failure, had died or defaulted on their treatment. High default rates have been reported to be the main cause of the growing number of treatment failure and increased drug resistance in retreatment cases. ${ }^{32}$

Our study results support previous reports of better success in treatment rate for females compared to males. Although this difference between males and females was not statistically significant, similar findings were reported in previous studies where men seemed to have more default rate compared to women. ${ }^{1,3,11,20}$ This study indicates that compared to patients residing in permanent housing, patients living in a shack or a traditional dwelling were found to be at greater risk for treatment failure, death and default. There has been an abundance of literature on poverty as a risk factor for TB adherence. ${ }^{3,4,6,33}$ Also, patients classified among the poorest and most socially marginalized struggle to afford transport to get to clinics for their TB treatment. It is expected that transport in the inner cities was generally easily accessible compared to rural areas but this may not be the case. Previous study findings in a systematic review assessing TB treatment compliance and the factors predictive for poor adherence among TB patients in sub-Saharan Africa have also reported the poor public transport as a problem that can result in high treatment default. ${ }^{20,25} \mathrm{An}$ unexpected finding was that patients who had a formal salary $(30.8 \%)$ experienced more treatment default, and death outcomes compared to those who either relied on family contributions $(28.9 \%)$ or social grants $(28.4 \%)$. It would have been expected that patients with a formal salary would be able to complete their treatment as they were expected to afford to get to the clinic as previous studies have also indicated a strong association between treatment default and low income. ${ }^{11,12}$

Similar to previous studies, ${ }^{12,}{ }^{19-21}$ we also found that retreatment was strongly associated with treatment default. With the Department of Health having introduced a programme of tracing TB treatment defaulters, it would help to determine the detailed reasons for 
defaulting even for patients who are retreated after previously defaulting. In this study self-reported reasons for defaulting from TB treatment included significant health improvement, structural and psychosocial including alcohol problems. Such information can be used to improve treatment adherence and lead to improvement in the overall TB epidemiological situation in the country. Of note was that specific clinics in the eThekwini health district had a substantially higher number of patients 39.1\% with treatment failure or default compared to the other two districts, Nelson Mandela and Siyanda (23.6\% and 20.8\% respectively). This finding needs further research and possibly increased health systems interventions in the eThekwini health district.

We found psychological distress was associated with treatment default, although in multivariate analysis this was not significant. This may be important to note as this sample being a vulnerable group, may require special attention in the control of TB. ${ }^{34}$ A study in India among new smear positive TB patients treated under DOTS found alcoholism as a predictor of noncompliance and poor patient provider interaction as a barrier to enhanced treatment compliance, ${ }^{35}$ the problem of alcohol use was also mentioned by self-report as a reason to default in this study. Contrary to the findings of previous studies where comorbid conditions resulted in treatment failure, ${ }^{36}$ it was encouraging that in the current study fewer participants $(26.4 \%)$ with three or more chronic conditions experienced treatment failure, default and death compared to those who had less or no other chronic conditions.

\section{Study limitations}

Caution should be taken when interpreting the results of this study because of certain limitations. One of the limitations was that most variables were assessed by self-report and desirable responses may have been given. Generalisability of our findings is limited to TB patients on treatment in public primary care facilities. Another limitation is the loss to follow-up (12\%) due to incorrect recording of names. The missing codebooks of fieldworkers and names not found in clinic registers may have resulted in the study findings being biased because those interviewed might differ from those not found, and it is possible that more non-traceable defaulters had died. Furthermore, the study only assessed TB patients in the urban and peri-urban health facilities.

\section{Conclusion}

We assessed the prevalence and associated factors of tuberculosis treatment failure, death and default among hazardous or harmful alcohol users in public health clinics. A high rate of treatment failure, death and default were found in the assessed TB patients. Several factors were identified including sociodemographic and being a retreatment patient that can guide interventions for the prevention of treatment failure, death and default.

\section{Acknowledgments}

The study was commissioned by the Department of Health in South Africa through a tender "NDOH: 21/2010-2011: Implementation and monitoring of screening and brief intervention for alcohol use disorders among tuberculosis patients" that was awarded to the Human Sciences Research Council. The analysis of this paper is partially based on the research supported by the National Research Foundation of South Africa (Grant reference number (UID) 85497). Opinions, findings and conclusions or recommendations expressed in any publication generated by the NRF supported research are those of the author(s), and that the NRF accepts no liability whatsoever in this regard.

\section{Competing interests}

The authors declare that they have no competing interests. 


\section{References}

1. Nahid P, Jarlsberg LG, Rudoy I, et al. Factors associated with mortality in patients with drug-susceptible pulmonary tuberculosis. BMC Infect Dis 2011;11:1.

2. Kim DH, Kim HJ, Park S, et al. Treatment outcomes and long-term survival in patients with extensively drug-resistant tuberculosis. Am J Respir Crit Care Med 2008;178:1075-1082.

3. Alavi SM, Salami N. The causes and risk factors of tuberculosis deaths in Khuzestan. Acta Medica Iranica 2009;47(2):89-92.

4. World Health Organization. The World Health Report 2002 - Global tuberculosis control, surveillance, planning, financing. Geneva: World Health Organization, 2002. http://www.who.int/tb/publications/2002/en/ index1.html (accessed 10 October 2012).

5. Aziz MA, Wright A, Laszlo A, et al. Epidemiology of antituberculosis drug resistance (The Global Project on Anti-tuberculosis Drug Resistance Surveillance): an updated analysis. Lancet 2006;368:2142-2154.

6. World Health Organization. The World Health Report 2010 - Global Tuberculosis Control Report. Geneva: World Health Organization, 2010. http://www.who. int/tb/publications/global_report/en/ (accessed 10 October 2012).

7. World Health Organisation. The World Health Report 2005 - Global Tuberculosis Control: Surveillance, Planning,Financing. Geneva:WorldHealth Organization, 2006. http://www.who.int/tb/publications/2005/en/ index.html (accessed 10 October 2012).

8. Kawai V, Soto G, Gilman RH, et al. Tuberculosis mortality, drug resistance, and infectiousness in patients with and without HIV infection in Peru. Am J Trop Med Hyg 2006;75(6):1027-1033.

9. Jianzhao H, Van den Hof S, Lin X, Yubang Q, Jinglong $\mathrm{H}$, Van der Werf MJ. Risk factors for non-cure among new sputum smear positive tuberculosis patients treated in tuberculosis dispensaries in Yunnan, China. BMC Health Serv Res 2011;11(1): 97.

10. Gandhi NR, Moll A, Sturm AW, et al. Extensively drug-resistant tuberculosis as a cause of death in patients co-infected with tuberculosis and HIV in a rural area of South Africa. Lancet 2006;368(9547):1575-1580.

11. Abuaku B, Tan H, Li X, Chen M, Huang X. Treatment default and death among tuberculosis patients in Hunan, China. Scand J Infect Dis 2010;42:281-287.

12. Finlay A, Lancaster J, Holtz TH, Weyer K, Miranda A, van der Walt M. Patient- and provider-level risk factors associated with default from tuberculosis treatment, South Africa, 2002: a case-control study. BMC Public Health 2012;12:56.

13. World Health Organisation. The World Health Report 2009 - Treatment of tuberculosis: guidelines for national programs. http://www.who.int/tb/ publications/tb_treatmentguidelines/en/index.html (accessed 28 October 2012).

14. Vree M, Huong NT, Duong BD E et al. Mortality and failure among tuberculosis patients who did not complete treatment in Vietnam: a cohort study Marleen. BMC Public Health 2007;7:134.

15. Khazaei HA, Rezaei N, Bagheri GR, et al. Epidemiology of tuberculosis in the southeastern Iran. Eur J Epidemiol 2005;20:879-883.

16. Solsona J, Cayla JA, Nadal J, et al. Screening for tuberculosis upon admission to shelters and free-meal services. Eur J Epidemiol 2001;17:123-128.

17. Slama K, Chiang C-Y, Enarson DA, et al. Tobacco and tuberculosis: a qualitative systematic review and meta-analysis. Int J Tuberc Lung Dis 2007;10:1049-61. 18. Lönnroth K, Jaramillo E, Williams BG, Dye C, Raviglione M. Drivers of tuberculosis epidemics: the role of risk factors and social determinants. Soc Sci Med 2009;68:2240-46.

19. Sanchez M, Bartholomay P, Arakaki-Sanchez D, et al. Outcomes of TB treatment by HIV status in national recording systems in Brazil, 2003-2008. PLoS One 2012;7(3):e33129.

20. Muture BN, Keraka MN, Kimuu PK, Kabiru EW, Ombeka VO, Oguya F. Factors associated with default from treatment among tuberculosis patients in Nairobi province, Kenya: a case control study. BMC Public Health 2011;11:696. 
21. Akpabio US, De Villiers JTP. A description of patients with recurrence of Pulmonary Tuberculosis in a Tuberculosis Hospital, Ermelo. Afr J Prm Health Care Fam Med 2011;3(1):Art. \#261, 8 pages. http:// dx.doi.org/10.4102/phcfm.v3il.261

22. Garrido MS, Penna ML, Perez-Porcuna TM, et al. Factors associated with tuberculosis treatment default in an endemic area of the Brazilian Amazon: a case control-study. PLoS One 2012;7(6):e39134.

23. World Health Organisation. The World Health Report 2007 - Global Tuberculosis Control. http:// www.who.int/tb/publications/global_report/2007/ pdf/key_findings.pdf (accessed 28 October 2012).

24. Munro SA, Lewin SA, Smith HJ, Engel ME, Fretheim

A, Volmink, J. Patient Adherence to Tuberculosis Treatment: A Systematic Review of Qualitative Research. PLoS Med 2007;4(7):e238.

25. Castelnuovo B. A review of compliance to anti tuberculosis treatment and risk factors for defaulting treatment in sub-Saharan Africa. Afr Health Sci 2010;10(4):320 - 324.

26. WHO, 2011. Non communicable diseases country profiles 2011. http://www.who.int/nmh/countries/ zaf_en.pdf (accessed 28 October 2012).

27. Centers for Disease Control and Prevention, 2011. Diseases \& Conditions. http://www.cdc.gov/ diseasesconditions/ (accessed 28 October 2012).

28. Kessler R, Andrews G, Colpe LJ, et al. Short screening scales to monitor population prevalences and trends in nonspecific psychological distress. Psychol
Med 2002, 32:959e976.

29. Kessler RC, Barker PR, Colpe LJ, et al. Screening for serious mental illness in the general population. Arch Gen Psychiatry 2003, 60(2):184e189.

30. Saunders JB, Aasland OG, Amundsen A, Grant M. Alcohol consumption and related problems among primary health care patients: WHO collaborative project on early detection of persons with harmful alcohol consumption. Addiction 1993; 88(3):349-362.

31. Reid MC, Fiellin DA, O'Connor PG. Harzardous and harmful alcohol consumption in primary care. Arch Intern Med 1999;159 (15):1681.

32. Department of Health: National tuberculosis management guidelines. Pretoria: Department of Health, 2009.

33. Jakubowiak WM, Bogorodskaya EM, Borisov ES, Danilova DI, Kourbatova EK. Risk factors associated with default among new pulmonary TB patients and social support in six Russion regions. Int J Tuberc Lung Dis 2007, 11(1): 46-53.

34. Figueroa-Munoza JI, Ramon-Pardob P. Tuberculosis control in vulnerable groups. Bulletin WHO 2008, 86 (9): 733-735

35. Singh V, Jaiswal A, Porter JDH, et al. TB control, poverty, and vulnerability in Delhi, India. Trop Med Int Health 2002, 7(8):693-700.

36. Vijay S, Kumar P, Chauhan LS, Vollepore BH, Kizhakkethil UP, Rao SG: Risk factors associated with default among new smear positive TB patients treated under DOTS in India. PloS ONE 2010, 5(4):e10043. 